$\xi=-\mathrm{g}$

\title{
An efficient scheme for solving a system of time- fractional order differential-algebraic equations by using fractional Laplace iteration method
}

\author{
Sameer Qasim Hasan ${ }^{1 *}$, Eman Mohammed Nemah ${ }^{2}$ \\ ${ }^{1}$ College of Education Almustansryah University \\ ${ }^{2}$ College of Education for Pure Sciencel Ibn Al-Haitham Baghdad University \\ *Corresponding author E-mail: dr.Sameer_Kasim@yahoo.com
}

\begin{abstract}
In this article, we propose an efficient algorithm for solving system of time- fractional differential-algebraic equations by using a fractional Laplace iteration method. The scheme is tested for some examples and the results demonstrate reliability and accuracy of this method.
\end{abstract}

Keywords: Riemann-Liouville Derivative; Analytic Solution; Fractional Laplace Iteration Method; Mittag-Leffller Functions; System of Time -Fractional Order Differential-Algebraic Equations.

\section{Introduction}

The fractional order calculus is a generalization of the integer order calculus to a real or complex number. Fractional derivative have been extensively investigated to their broad applications in mathematics, physics and engineering [1-3]. A review of some applications of fractional calculus in continuum and statistical mechanics is given by Mainardi [4]. Recently, many important mathematical models can be expressed in term of system of differential-algebraic equations. The exact solution of most of the differential-algebraic equations (FDEs) cannot be found easily, and this has mandated the use of both analytical and numerical method. In recent years, many researchers have focused on the numerical solution of fractional differential -algebraic equations. Some numerical methods have been developed, such as implicit RungeKutta method [5], Padé approximation method [6-9], homotopy perturbation method [10-14], Adomian decomposition method [15-19], homotopy analysis method [20-21], variation iteration method [22-24], homotopy analysis transform method [25].

In 2013, Habibolla et al. [26] presented an alternative approach based on Laplace iterative method (LIM) for finding series solutions to linear and nonlinear systems of PDEs. The applied method gave rapidly convergent successive approximations.

In our article, we have use Fractional Laplace Iteration Method (FLIM) successfully to find the approximate analytical solution linear homogeneous, non-homogeneous FDAEs with time-and space fractional derivatives. These problems have not yet been solved by any researcher.

The rest of this paper is organized as follows. In section 2, we give the some necessary definitions and mathematical preliminaries of the fractional calculus theory. In section 3, we give analysis of the method used. In section 4 , the proposed method is applied to several examples. Also a conclusion is given in the last section.

\section{Preliminaries and notations}

In this section, we give some basic definitions and properties of fractional calculus theory which will be used in this paper.

Definition 2.1: [27] A real function $f(t), t>0$, is said to be in the space $C_{\mu}, \mu \in R$ if there exists a real number $p>\mu$, such that $f(t)=t^{p} f_{1}(t)$, where $f_{1}(t) \in C[0, \infty)$ and it is said to be in the space $C_{\mu}^{m}$ if and only if $f^{(m)} \in C_{\mu}, m \in N$.

Definition 2.2: The Riemann-Liouville fractional order integral of $\alpha>0$ of function $f(t) \in C_{\mu}, \mu>-1$ is defined as [27]:

$J^{\alpha} f(t)=\frac{1}{\Gamma(\alpha)} \int_{0}^{t}(t-\tau)^{\alpha-1} f(\tau) d \tau$

$J^{0} f(t)=f(t)$

Definition 2.3: The fractional derivative of function $f(t)$ in $\mathrm{Ca}$ puto sense is defined as [27]:

${ }_{\mathrm{RL}} \mathrm{D}^{\alpha} \mathrm{f}(\mathrm{t})=\mathrm{J}^{\mathrm{m}-\alpha} \mathrm{D}^{\mathrm{m}} \mathrm{f}(\mathrm{t})=\frac{1}{\Gamma(\mathrm{m}-\alpha)} \frac{\mathrm{d}^{\mathrm{m}}}{\mathrm{dt}^{\mathrm{m}}} \int_{0}^{\mathrm{t}}(\mathrm{t}-$

$\tau)^{m-\alpha-1} f(\tau) d \tau, t>0$

For $\mathrm{m}-1<\alpha \leq \mathrm{m}, \mathrm{m} \in \mathrm{N}, \mathrm{t}>0, \mathrm{f} \in \mathrm{C}_{-1}^{\mathrm{m}}$.

Definition 2.4: [28] The single parameter and the two parameters variants of the Mittag-Leffler function are denoted by $E_{\alpha}(t)$ and $E_{\alpha, \beta}(t)$, respectively, which are relevant for their connection with fractional calculus, and are defined as:

$\mathrm{E}_{\propto}(\mathrm{t})=\sum_{\mathrm{k}=0}^{\infty} \frac{\mathrm{t}^{\mathrm{k}}}{\Gamma(\propto \mathrm{k}+1)}, \propto>0, \mathrm{t} \in \mathrm{C}$ 


$$
\mathrm{E}_{\propto, \beta}(\mathrm{t})=\sum_{\mathrm{k}=0}^{\infty} \frac{\mathrm{t}^{\mathrm{k}}}{\Gamma(\alpha \mathrm{k}+\beta)}, \propto, \beta>0, \mathrm{t} \in \mathrm{C}
$$

For special choices of the values of the parameter $\alpha, \beta$ we obtain well-known classical functions, e.g.:

$E_{1}(t)=e^{t}, E_{\propto, 1}(t)=E_{\alpha}(t)$

As we will see later, classical derivatives of the Mittag-Leffler function appear in solution of FDEs. Since the series (2.4) is uniformly convergent we may differentiate term by term and obtain

$$
\mathrm{E}^{(\mathrm{m})}{ }_{\alpha, \beta}(\mathrm{t})=\sum_{\mathrm{k}=0}^{\infty} \frac{(\mathrm{k}+\mathrm{m}) !}{\mathrm{k} !} \frac{\mathrm{t}^{\mathrm{k}}}{\Gamma(\alpha \mathrm{k}+\alpha \mathrm{m}+\beta)}
$$

Lemma 2.5 [28]: For $\alpha, \beta>0$ and $s^{\alpha}>|a|$ we have the following inverse Laplace transforms formula

$$
\mathcal{L}^{-1}\left(\frac{s^{\alpha-\beta}}{s^{\alpha}+a}\right)=t^{\beta-1} E_{\alpha, \beta}\left(-a t^{\alpha}\right)
$$

Definition 2.6: The Laplace transform $\mathcal{L}[x(t)]$ of the RiemannLiouville fractional derivative is given as [24]:

$$
\mathcal{L}\left[{ }_{R L} D^{\alpha} f(t)\right]=s^{\alpha} \mathcal{L}[f(t)]-\left.\sum_{k=0}^{m-1} s^{k}\left({ }_{R L} D^{\alpha-k-1} f(t)\right)\right|_{t=0}
$$

Recall the Laplace transform of R-L derivative for $0<\alpha \leq 1$ is:

$$
\mathcal{L}\left[{ }_{R L} D^{\alpha} f(t)\right]=s^{\alpha} \mathcal{L}[f(t)]-\left.\left({ }_{R L} D^{\alpha-1} f(t)\right)\right|_{t=0}
$$

Property 2.7: [27] The compositions of Riemann-Liouville derivative operators ${ }_{R L} D^{\alpha} x(t)$ and ${ }_{R L} D^{\beta} x(t)$ are as follows:

$$
\begin{aligned}
& { }_{R L} D^{\alpha}\left({ }_{R L} D^{\beta} x(t)\right)={ }_{R L} D^{\alpha+\beta} x(t)- \\
& \left.\sum_{j=0}^{n}\left({ }_{R L} D^{\beta-j} x(t)\right)\right|_{t=0} \frac{t^{-\alpha-j}}{\Gamma(1-\alpha-j)}
\end{aligned}
$$

And

$$
\begin{aligned}
& { }_{R L} D^{\beta}\left({ }_{R L} D^{\alpha} x(t)\right)={ }_{R L} D^{\alpha+\beta} x(t)- \\
& \left.\sum_{j=0}^{m}\left({ }_{R L} D^{\alpha-j} x(t)\right)\right|_{t=0} \frac{t^{-\beta-j}}{\Gamma(1-\beta-j)}
\end{aligned}
$$

Where $\mathrm{m}-1<\alpha \leq \mathrm{m}, \mathrm{n}-1<\beta \leq \mathrm{n}$ and $\mathrm{m}, \mathrm{n} \in \mathrm{N}$.

\section{Analysis of the FLIM}

In this article, we consider the following non-homogenous, nonlinear system of fractional order differential-algebraic equations

$$
\left.\begin{array}{c}
{ }_{\mathrm{RL}} \mathrm{D}^{\alpha_{\mathrm{i}}}\left({ }_{\mathrm{RL}} \mathrm{D}^{\beta_{i}} \mathrm{x}_{\mathrm{i}}(\mathrm{t})\right)+\mathrm{a}_{\mathrm{i}} \mathrm{x}_{\mathrm{i}}(\mathrm{t})=\mathrm{h}_{\mathrm{i}}\left(\mathrm{t}, \mathrm{x}_{1}, \mathrm{x}_{2}, \ldots, \mathrm{x}_{\mathrm{n}}\right) \\
0=\mathrm{g}\left(\mathrm{t}, \mathrm{x}_{1}, \mathrm{x}_{2}, \ldots, \mathrm{x}_{\mathrm{n}}\right)
\end{array}\right\}
$$

With initial conditions

$$
\begin{aligned}
& x_{i}(0)=b_{i}, x_{1}(0)=c_{i},\left.\left({ }_{R L} D^{\beta_{i}-1} x(t)\right)\right|_{t=0}= \\
& d_{i},\left.\left(R_{R L} D^{\alpha_{i}+\beta_{i}-1} x(t)\right)\right|_{t=0}=e_{i} i=1,2, \ldots, n-1, x_{n}(0)=s_{i}
\end{aligned}
$$

Here $a_{i}, b_{i}, c_{i}, d_{i}, e_{i}, s_{i}$ are constants and ${ }_{R L} D^{\alpha_{i}}\left({ }_{R L} D^{\beta_{i}} x(t)\right)$ is the compositions of Riemann - Liouville derivative operators satisfying the relation $0<\alpha_{i}, \beta_{i}<1$. Eq. (3.1) can be rewritten as:

$$
\left.\begin{array}{c}
\mathrm{L}_{1} \mathrm{x}_{1}(\mathrm{t})+\mathrm{N}_{1}\left(\mathrm{t}, \mathrm{x}_{1}, \mathrm{x}_{2}, \ldots, \mathrm{x}_{\mathrm{n}}\right)=\mathrm{f}_{1}(\mathrm{t}) \\
\mathrm{L}_{2} \mathrm{x}_{2}(\mathrm{t})+\mathrm{N}_{2}\left(\mathrm{t}, \mathrm{x}_{1}, \mathrm{x}_{2}, \ldots, \mathrm{x}_{\mathrm{n}}\right)=\mathrm{f}_{2}(\mathrm{t}) \\
\cdot \\
\cdot \\
\mathrm{L}_{\mathrm{n}-1} \mathrm{x}_{\mathrm{n}-1}(\mathrm{t})+\mathrm{N}_{\mathrm{n}}\left(\mathrm{t}, \mathrm{x}_{1}, \mathrm{x}_{2}, \ldots, \mathrm{x}_{\mathrm{n}}\right)=\mathrm{f}_{\mathrm{n}-1}(\mathrm{t}) \\
0=\mathrm{g}\left(\mathrm{t}, \mathrm{x}_{1}, \mathrm{x}_{2}, \ldots, \mathrm{x}_{\mathrm{n}}\right)
\end{array}\right\}
$$

Where $L_{i}$ is a linear operator, $N_{i}$ a nonlinear operator and $f_{i}(t)$ is a nonhomogeneous item form $\mathrm{i}=1,2 \ldots \mathrm{n}-1$. Eq. (3.2) can be rewritten down as a correction function in the following way:

$\mathrm{L}_{\mathrm{i}} \mathrm{x}_{\mathrm{i}}(\mathrm{t})=\mathrm{f}_{\mathrm{i}}(\mathrm{t})-\mathrm{N}_{\mathrm{i}}\left(\mathrm{t}, \mathrm{x}_{1}, \mathrm{x}_{2}, \ldots, \mathrm{x}_{\mathrm{n}}\right)=\mathrm{R}_{\mathrm{i}}\left(\mathrm{t}, \mathrm{x}_{1}, \mathrm{x}_{2}, \ldots, \mathrm{x}_{\mathrm{n}}\right)$,

$\mathrm{i}=1,2 \ldots \mathrm{n}-1$

Therefore:

$\mathrm{L}_{\mathrm{i}} \mathrm{x}_{\mathrm{i}}(\mathrm{t})=\mathrm{R}_{\mathrm{i}}\left(\mathrm{t}, \mathrm{x}_{1}, \mathrm{x}_{2}, \ldots, \mathrm{x}_{\mathrm{n}}\right), \mathrm{i}=1,2 \ldots \mathrm{n}-1$

The Fractional Laplace Iteration Method assumed a series solution for $\mathrm{x}_{\mathrm{i}}$ given by an infinite sum of components:

$x_{i}(t)=\lim _{n \rightarrow \infty} x_{i}^{p}(t)=\lim _{n \rightarrow \infty} \sum_{j=0}^{p} v_{i}^{j}(t)$,

$\mathrm{i}=1,2, \ldots, \mathrm{n}-1$

In which $x_{i}^{n}$ indicates the $n$-th approximation of $x_{i}$, where $v_{i}^{j}$ is the $\mathrm{j}^{\text {th }}$ component of the solution of $\mathrm{x}_{\mathrm{i}}$ and $\mathrm{v}_{\mathrm{i}}^{0}$ is the solution of $\mathrm{L}_{\mathrm{i}} \mathrm{x}_{\mathrm{i}}=$ 0 , along with the following initial conditions of the main problem:

$\mathrm{v}_{\mathrm{i}}^{1}(\mathrm{t})=\varphi_{\mathrm{i}}\left(\mathrm{v}_{\mathrm{i}}^{0}\right)$

$\mathrm{v}_{\mathrm{i}}^{\mathrm{k}+1}(\mathrm{t})=\varphi_{\mathrm{i}}\left(\sum_{\mathrm{j}=0}^{\mathrm{k}} \mathrm{v}_{\mathrm{i}}^{\mathrm{j}}(\mathrm{t})\right)-\sum_{\mathrm{j}=0}^{\mathrm{k}} \mathrm{v}_{\mathrm{i}}^{\mathrm{j}}(\mathrm{t}), \mathrm{k} \geq 1$

In which $\varphi_{i}\left(v_{i}^{k}\right)$ is obtained as follows:

$\mathrm{L}_{\mathrm{i}} \varphi_{\mathrm{i}}\left(\mathrm{v}_{1}^{\mathrm{k}}, \mathrm{v}_{2}^{\mathrm{k}}, \ldots, \mathrm{v}_{\mathrm{n}}^{\mathrm{k}}\right)=\mathrm{R}_{\mathrm{i}}\left(\mathrm{t}, \mathrm{x}_{1}, \mathrm{x}_{2}, \ldots, \mathrm{x}_{\mathrm{n}}\right)$

Using the homogenous initial conditions, supposing that $\mathrm{L}_{\mathrm{i}}$ linear operator, therefore, taking Laplace transform to both sides of Eq. (3.5) in the usual way and using the homogenous initial conditions, the result can be obtained as following:

$\mathrm{p}_{\mathrm{i}}(\mathrm{s}) \cdot \Phi_{\mathrm{i}}^{\mathrm{k}}(\mathrm{s})=\mathcal{R}_{\mathrm{i}}\left(\mathrm{v}_{\mathrm{i}}^{\mathrm{k}}(\mathrm{s})\right)$,

Where $\mathcal{L}\left[\varphi_{\mathrm{i}}\left(\mathrm{v}_{1}^{\mathrm{k}}, \mathrm{v}_{2}^{\mathrm{k}}, \ldots, \mathrm{v}_{\mathrm{n}}^{\mathrm{k}}\right)\right]=\Phi_{\mathrm{i}}^{\mathrm{k}}, \mathrm{p}_{\mathrm{i}}(\mathrm{s})$ is a fractional polynomial with the fractional degree of the highest derivative in Eq. (3.6) (The same as the highest order of the linear operator $\mathrm{L}_{\mathrm{i}}$ ). Thus,

$\mathcal{L}[\mathrm{w}]=\varpi, \psi_{\mathrm{i}}(\mathrm{s})=\frac{1}{\mathrm{p}_{\mathrm{i}}(\mathrm{s})}, \mathcal{L}\left[\mathrm{u}_{\mathrm{i}}(\mathrm{t})\right]=\psi_{\mathrm{i}}(\mathrm{s})$

In Equations (3.5) and (3.6), the function $\mathcal{R}_{\mathrm{i}}\left(\mathrm{v}_{\mathrm{i}}^{\mathrm{k}}(\mathrm{s})\right.$ ) and $\mathrm{R}_{\mathrm{i}}\left(\mathrm{t}, \mathrm{x}_{1}, \mathrm{x}_{2}, \ldots, \mathrm{x}_{\mathrm{n}}\right)$ are abbreviated as $\mathcal{R}_{\mathrm{i}}$ and $\mathrm{R}_{\mathrm{i}}$ respectively. Hence, Eq. (3.6) is rewritten as:

$\Phi_{\mathrm{i}}^{\mathrm{k}}(\mathrm{s})=\mathcal{R}_{\mathrm{i}}\left(\left(\mathrm{v}_{1}^{\mathrm{k}}, \mathrm{v}_{2}^{\mathrm{k}}, \ldots, \mathrm{v}_{\mathrm{n}}^{\mathrm{k}}\right)(\mathrm{s})\right) \cdot \Psi_{\mathrm{i}}(\mathrm{s})$

Now, by applying the inverse Laplace Transform to both side of Eq. (3.8) and using the convolution Theorem, the following relation can be presented:

$\emptyset_{i}\left(v_{1}^{k}, v_{2}^{k}, \ldots, v_{n}^{k}\right)=\int_{0}^{t} R_{i}\left(\left(v_{1}^{k}, v_{2}^{k}, \ldots, v_{n}^{k}\right)(\tau)\right) \cdot u_{i}(t-\tau) d \tau$

Therefore

$x_{i}^{p+1}(t)=\sum_{j=0}^{p+1} v_{i}^{j}(t)=x_{i}^{0}(t)+\int_{0}^{t} R_{i}\left(x_{i}^{p}(\tau)\right) \cdot u_{i}(t-\tau) d \tau$, $i=1,2, \ldots, n-1$

After identifying the initial approximation of $x_{i}^{0}$, the remaining approximations $x_{i}^{p}, p>0$ can be determined so that each term can be determined by previous terms and the approximation of iteration formula can be entirely evaluated.

Consequently, the exact solution may be obtained by:

$x_{i}=\lim _{p \rightarrow \infty} x_{i}^{p}(t)=\lim _{p \rightarrow \infty} \sum_{j=0}^{p} v_{i}^{j}(t)$ 
Which is the Fractional Laplace Iteration method.

\section{Numerical experiments}

In this part, we introduce some applications on FLIM to solve differential- algebraic equations with time- fractional derivatives:

Example 1: consider the following non-homogenous system of linear space-time fractional order differential -algebraic equations:

$$
\left.\begin{array}{c}
{ }_{R L} D^{\alpha}\left({ }_{R L} D^{\beta} x(t)\right)+x(t)-y(t)=-\sin t, \\
x(t)+y(t)=\sin t+\cos t, t \in[0,1], 0<\alpha, \beta<1
\end{array}\right\}
$$

Subject to initial conditions $\mathrm{x}(0)=1, \mathrm{y}(0)=0, \dot{x}(0)=0$,

$$
\left.\left({ }_{R L} D^{\beta-1} x(t)\right)\right|_{t=0}=a,\left.\left({ }_{R L} D^{\alpha+\beta-1} x(t)\right)\right|_{t=0}=b
$$

For the special case $\alpha=\beta=1$, we have analytical solution $x(t)=\cos t, y(t)=\sin t$.

\section{Solution:}

From the Eq. (4.1), optimal selection auxiliary linear operator the equation is represented as follows:

$L x(t):{ }_{R L} D^{\alpha}\left({ }_{R L} D^{\beta} x(t)\right)+x(t)$

Therefore $\emptyset\left(v_{1}^{k}, v_{2}^{k}\right)$ is defined as:

$\emptyset\left(v_{1}^{k}, v_{2}^{k}\right)=\int_{0}^{t} u(t-\tau)\left[v_{2}^{k}(\tau)-\sin \tau\right] d \tau$

Then, using Eq. (4.1), the fractional Laplace iteration method in tdirection for the calculation of the approximate solution of Eq. (4.2) can be readily obtained as:

$$
\left.\begin{array}{c}
x_{n+1}(t)=x_{0}(t)+\int_{0}^{t} u(t-\tau)\left[y_{n}(\tau)-\sin \tau\right] d \tau, \\
y_{n}(t)=x_{n}(t)+\sin t-\cos t
\end{array}\right\}
$$

Case 1: $\alpha+\beta<1\left(\right.$ if $\left.\alpha=\frac{1}{5}, \beta=\frac{1}{4}\right)$

$$
\begin{aligned}
& L x(t):{ }_{R L} D^{\frac{1}{5}}\left({ }_{R L} D^{\frac{1}{4}} x(t)\right)+x(t) \\
& \Rightarrow p(s)=s^{\frac{9}{20}}+1 \\
& \Rightarrow \psi(s)=\frac{1}{p(s)}=\frac{1}{s^{\frac{9}{20}}+1} \\
& \Rightarrow u(t)=\mathcal{L}^{-1}[\psi(s)]=t^{\frac{-11}{20}} E_{\frac{9}{20}, \frac{9}{20}}\left(-t^{\frac{9}{20}}\right)
\end{aligned}
$$

Where the initial approximation must be satisfied by the following equations:

$$
\begin{aligned}
& L x(t)=0, x(0)=1,\left.\left({ }_{R L} D^{\beta-1} x(t)\right)\right|_{t=0}=\left.\left({ }_{R L} D^{\frac{-3}{4}} x(t)\right)\right|_{t=0}=0 \\
& \left.\left({ }_{R L} D^{\alpha+\beta-1} x(t)\right)\right|_{t=0}=\left.\left({ }_{R L} D^{\frac{-11}{20}} x(t)\right)\right|_{t=0}=0, \\
& x_{0}(t)=E_{\frac{9}{20^{\prime}}}\left(-t^{\frac{9}{20}}\right) \\
& y_{0}(t)=x_{0}(t)+\sin t-\cos t \\
& =E_{\frac{9}{20^{\prime}}, 1}\left(-t^{\frac{9}{20}}\right)+\sin t-\cos t
\end{aligned}
$$

Accordingly, by Eq. (4.3) the higher order approximation of the exact solution can be obtained as follows: $x_{1}(t)=\sum_{k=0}^{\infty} \frac{\left(-t^{\frac{9}{20}}\right)^{k}}{\Gamma\left(\frac{9}{20} k+1\right)}+$

$\int_{0}^{\tau}\left[\left((t-\tau)^{\frac{-11}{20}} \sum_{k=0}^{\infty} \frac{\left(-(t-\tau)^{\frac{9}{20}}\right)^{k}}{\Gamma\left(\frac{9}{20} k+\frac{9}{20}\right)}\right)\left(\sum_{k=0}^{\infty} \frac{\left(-\tau^{\frac{9}{20}}\right)^{k}}{\Gamma\left(\frac{9}{20} k+1\right)}-\cos \tau\right)\right] d \tau$

$y_{1}(t)=\sum_{k=0}^{\infty} \frac{\left(-t^{\frac{9}{20}}\right)^{k}}{\Gamma\left(\frac{9}{20} k+1\right)}+$

$\int_{0}^{\tau}\left[\left((t-\tau)^{\frac{-11}{20}} \sum_{k=0}^{\infty} \frac{\left(-(t-\tau)^{\frac{9}{20}}\right)^{k}}{\Gamma\left(\frac{9}{20} k+\frac{9}{20}\right)}\right)\left(\sum_{k=0}^{\infty} \frac{\left(-\tau^{\frac{9}{20}}\right)^{k}}{\Gamma\left(\frac{9}{20} k+1\right)}-\cos \tau\right)\right] d \tau+$ $\sin t-\cos t$

Case 2: $\alpha+\beta=1\left(\right.$ if $\left.\alpha=\frac{3}{10}, \beta=\frac{7}{10}\right)$

$L x(t):{ }_{R L} D^{\frac{3}{10}}\left({ }_{R L} D^{\frac{7}{10}} x(t)\right)+x(t)$

$\Rightarrow p(s)=s+1$

$\Rightarrow \psi(s)=\frac{1}{p(s)}=\frac{1}{s+1}$

$\Rightarrow u(t)=\mathcal{L}^{-1}[\psi(s)]=e^{-t}$

Where the initial approximation must be satisfied by the following equations:

$$
\begin{aligned}
& L x(t)=0, x(0)=1,\left.\left({ }_{R L} D^{\beta-1} x(t)\right)\right|_{t=0}=\left.\left({ }_{R L} D^{\frac{-3}{10}} x(t)\right)\right|_{t=0}=0 \\
& \Rightarrow x_{0}(t)=E_{1,1}(-t)=e^{-t} \\
& y_{0}(t)=x_{0}(t)+\sin t-\cos t \\
& =e^{-t}+\sin t-\cos t
\end{aligned}
$$

Accordingly, by Eq. (4.3) the higher order approximation of the exact solution can be obtained as follows:

$x_{1}(t)=\sum_{k=0}^{\infty} \frac{(-t)^{k}}{\Gamma(k+1)}+\int_{0}^{\tau}\left[\left(\sum_{k=0}^{\infty} \frac{(\tau-t)^{k}}{\Gamma(k+1)}\right)\left(\sum_{k=0}^{\infty} \frac{(-\tau)^{k}}{\Gamma(k+1)}-\right.\right.$ $\cos \tau)] d \tau$

$y_{1}(t)=\sum_{k=0}^{\infty} \frac{(-t)^{k}}{\Gamma(k+1)}+\int_{0}^{\tau}\left[\left(\sum_{k=0}^{\infty} \frac{(\tau-t)^{k}}{\Gamma(k+1)}\right)\left(\sum_{k=0}^{\infty} \frac{(-\tau)^{k}}{\Gamma(k+1)}-\right.\right.$ $\cos \tau)] d \tau+\sin t-\cos t$

Case 3: $\alpha+\beta>1\left(\right.$ if $\left.\alpha=\frac{1}{2}, \beta=\frac{2}{3}\right)$

$$
\begin{aligned}
& L x(t):{ }_{R L} D^{\frac{1}{2}}\left({ }_{R L} D^{\frac{2}{3}} x(t)\right)+x(t) \\
& \Rightarrow p(s)=s^{\frac{7}{6}}+1 \\
& \Rightarrow \psi(s)=\frac{1}{p(s)}=\frac{1}{s^{\frac{7}{6}}+1} \\
& \Rightarrow u(t)=\mathcal{L}^{-1}[\psi(s)]=t^{\frac{1}{6} E_{\frac{7}{6} \cdot \frac{7}{6}}\left(-t^{\frac{7}{6}}\right)}
\end{aligned}
$$

Where the initial approximation must be satisfied by the following equations: 


$$
\begin{aligned}
& L x(t)=0, x(0)=1, \dot{x}(0)=0,\left.\left({ }_{R L} D^{\beta-1} x(t)\right)\right|_{t=0}= \\
& \left.\left({ }_{R L} D^{\frac{-1}{3}} x(t)\right)\right|_{t=0}=0,\left.\left({ }_{R L} D^{\alpha+\beta-1} x(t)\right)\right|_{t=0}=\left.\left({ }_{R L} D^{\frac{1}{6}} x(t)\right)\right|_{t=0}= \\
& 0, \Rightarrow x_{0}(t)=E_{\frac{7}{6}, 1}\left(-t^{\frac{7}{6}}\right) \\
& y_{0}(t)=x_{0}(t)+\sin t-\cos t \\
& \quad=E_{\frac{7}{6}, 1}\left(-t^{\frac{7}{6}}\right)+\sin t-\cos t
\end{aligned}
$$

Accordingly, by Eq. (4.3) the higher order approximation of the exact solution can be obtained as follows:

$$
\begin{aligned}
& x_{1}(t)=\sum_{k=0}^{\infty} \frac{\left(-t^{\frac{7}{6}}\right)^{k}}{\Gamma\left(\frac{7}{6} k+1\right)}+ \\
& \int_{0}^{\tau}\left[\left((t-\tau)^{\frac{1}{6}} \sum_{k=0}^{\infty} \frac{\left(-(t-\tau)^{\frac{7}{6}}\right)^{k}}{\Gamma\left(\frac{7}{6} k+\frac{7}{6}\right)}\right)\left(\sum_{k=0}^{\infty} \frac{\left(-\tau^{\frac{7}{6}}\right)^{k}}{\Gamma\left(\frac{7}{6} k+1\right)}-\cos \tau\right)\right] d \tau
\end{aligned}
$$$$
y_{1}(t)=\sum_{k=0}^{\infty} \frac{\left(-t^{\frac{7}{6}}\right)^{k}}{\Gamma\left(\frac{7}{6} k+1\right)}+
$$$$
\int_{0}^{\tau}\left[\left((t-\tau)^{\frac{1}{6}} \sum_{k=0}^{\infty} \frac{\left(-(t-\tau)^{\frac{7}{6}}\right)^{k}}{\Gamma\left(\frac{7}{6} k+\frac{7}{6}\right)}\right)\left(\sum_{k=0}^{\infty} \frac{\left(-\tau^{\frac{7}{6}}\right)^{k}}{\Gamma\left(\frac{7}{6} k+1\right)}-\cos \tau\right)\right] d \tau+
$$

$\sin t-\cos t$

Table 1: Numerical Results of the Solution in Example 1

\begin{tabular}{llllll}
\hline $\mathrm{T}$ & \multicolumn{1}{c}{$x_{1}$} & \multicolumn{1}{c}{$x_{1}$} & \multicolumn{1}{c}{$x_{1}$} & \\
& $\alpha=\frac{1}{5}, \beta=\frac{1}{4}$ & $\alpha=\frac{3}{10}, \beta=\frac{7}{10}$ & $\alpha=\frac{1}{2}, \beta=\frac{2}{3}$ & Exact solution \\
\hline 0 & 1 & 1 & 1 & 1 \\
0.1 & 0.622 & 0.9 & 0.939 & 0.995 \\
0.2 & 0.507 & 0.802 & 0.868 & 0.98 \\
0.3 & 0.421 & 0.708 & 0.797 & 0.955 \\
0.4 & 0.36 & 0.613 & 0.729 & 0.921 \\
0.5 & 0.313 & 0.534 & 0.666 & 0.878 \\
0.6 & 0.276 & 0.45 & 0.611 & 0.825 \\
0.7 & 0.249 & 0.387 & 0.563 & 0.765 \\
0.8 & 0.231 & 0.325 & 0.523 & 0.697 \\
0.9 & 0.221 & 0.27 & 0.492 & 0.622 \\
1 & 0.22 & 0.224 & 0.471 & 0.54 \\
\hline
\end{tabular}

Table 1 shows the approximate solutions for Eq. (4.3) obtained for different values of $\alpha$ and $\beta$ using our method. The results are in good agreement with the results of the exact solutions.

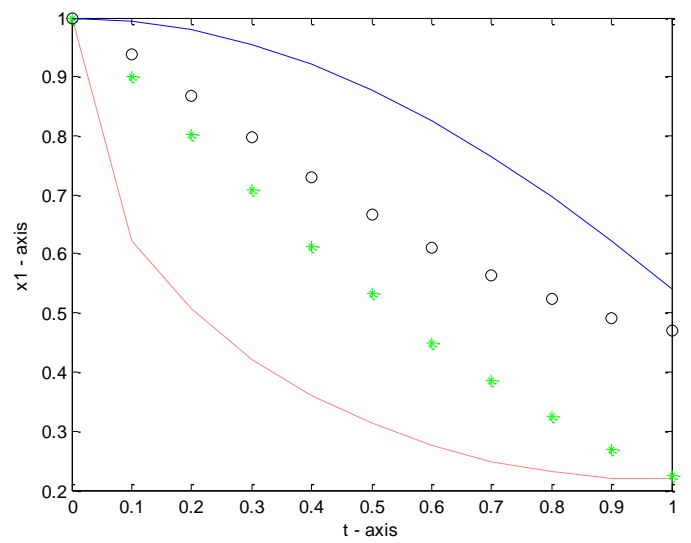

Fig. 1: The red dotted line (...) is the approximate solution when $\alpha=$ $\frac{1}{5}, \beta=\frac{1}{4}(\alpha+\beta<1)$, the green asterisks (***) is the approximate solution when $\alpha=\frac{3}{10}, \beta=\frac{7}{10}(\alpha+\beta=1$ ), the black circles (ooo) is the approximate solution when $\alpha=\frac{1}{2}, \beta=\frac{2}{3}(\alpha+\beta>1)$, and the Continuous Line ( --) is the Exact Solution.
Example 2: consider the following homogenous system of linear space-time fractional order differential -algebraic equations:

$$
\left.\begin{array}{c}
{ }_{R L} D^{\alpha}\left({ }_{R L} D^{\beta} x(t)\right)+x(t)-2 t y ́(t)+2 \dot{x}(t)+2 t \grave{z}(t)- \\
(4+2 t) y(t)+\left(2 t^{2}+6 t\right) z(t)=0, \\
{ }_{R L} D^{\alpha}\left({ }_{R L} D^{\beta} y(t)\right)-\dot{z}(t)-y(t)+2 t z(t)=0, \\
z(t)-\sin t=0, t \in[o, 1], 0<\alpha, \beta<1
\end{array}\right\}
$$

Subject to initial conditions $\mathrm{x}(0)=\mathrm{y}(0)=1, \mathrm{z}(0)=0, \dot{x}(0)=0$,

$y(0)=1,\left.\left({ }_{R L} D^{\alpha+\beta-1} x(t)\right)\right|_{t=0}=a$,

$\left.\left({ }_{R L} D^{\beta-1} x(t)\right)\right|_{t=0}=b,\left.\left(D^{\alpha+\beta-1} y(t)\right)\right|_{t=0}=$

$c,\left.\left({ }_{R L} D^{\beta-1} y(t)\right)\right|_{t=0}=d$.

For the special case $\alpha=\beta=1$, we have analytical solution

$x(t)=e^{-t}+t e^{t}, y(t)=e^{t}+t \sin t$, and $\mathrm{z}(\mathrm{t})=\sin t$

\section{Solution:}

From the Eq. (4.4), optimal selection auxiliary linear operator the equation is represented as follows:

$L_{1} x(t):{ }_{R L} D^{\alpha}\left({ }_{R L} D^{\beta} x(t)\right)+x(t)$

$L_{2} y(t):{ }_{R L} D^{\alpha}\left({ }_{R L} D^{\beta} x(t)\right)-y(t)$

Therefore $\emptyset_{i}\left(v_{1}^{k}, v_{2}^{k}, v_{3}^{k}\right), \mathrm{i}=1,2$; are defined as:

$$
\left.\begin{array}{l}
\emptyset_{1}\left(v_{1}^{k}, v_{2}^{k}, v_{3}^{k}\right)=\int_{0}^{t} u_{1}(t-\tau)\left[2 \tau\left(v_{2}^{k^{\prime}}(\tau)\right)-2\left(v_{1}^{k}(\tau)\right)\right. \\
\left.-2 \tau\left(v_{3}^{k}(\tau)\right)+(4+2 \tau) v_{2}^{k}(\tau)-\left(2 \tau^{2}+6 \tau\right) v_{3}^{k}(\tau)\right] d \tau \\
\emptyset_{2}\left(v_{1}^{k}, v_{2}^{k}, v_{3}^{k}\right)=\int_{0}^{t} u_{2}(t-\tau)\left[\left(v_{3}^{k}(\tau)\right)-2 \tau v_{3}^{k}(\tau)\right] d \tau
\end{array}\right\}
$$

Then, using Eq. (4.4), the fractional Laplace iteration method in tdirection for the calculation of the approximate solution of Eq. (4.5) can be readily obtained as:

$$
\left.\begin{array}{c}
x_{n+1}(t)=x_{0}(t)+\int_{0}^{t} u_{1}(t-\tau)\left[2 \tau\left(y_{n}^{\prime}(\tau)\right)-\right. \\
2\left(x_{n}^{\prime}(\tau)\right)-2 \tau\left(z_{n}^{\prime}(\tau)\right)+(4+2 \tau)\left(y_{n}^{\prime}(\tau)\right) \\
\left.-\left(2 \tau^{2}+6 \tau\right) z_{n}(\tau)\right] d \tau \\
y_{n+1}(t)=z_{0}(t)+\int_{0}^{t} u_{2}(t-\tau)\left[\left(z_{n}^{\prime}(\tau)\right)-2 \tau z_{n}(\tau)\right] d \tau, \\
z_{n}=\sin t
\end{array}\right\}
$$

Case 1: $\alpha+\beta<1\left(\right.$ if $\left.\alpha=\frac{1}{2}, \beta=\frac{1}{3}\right)$

$$
\begin{aligned}
& L_{1} x(t):{ }_{R L} D^{\frac{1}{2}}\left({ }_{R L} D^{\frac{1}{3}} x(t)\right)+x(t) \\
& \Rightarrow p_{1}(s)=s^{\frac{5}{6}}+1 \\
& \Rightarrow \psi_{1}(s)=\frac{1}{p_{1}(s)}=\frac{1}{s^{\frac{5}{6}}+1} \\
& \Rightarrow u_{1}(t)=\mathcal{L}^{-1}\left[\psi_{1}(s)\right]=t^{\frac{-1}{6}} E_{\frac{5}{6^{\prime}} \frac{5}{6}}\left(-t^{\frac{5}{6}}\right)
\end{aligned}
$$

And

$$
\begin{aligned}
& L_{2} Z(t):{ }_{R L} D^{\frac{1}{2}}\left({ }_{R L} D^{\frac{1}{3}} y(t)\right)-y(t) \\
& \Rightarrow p_{2}(s)=s^{\frac{5}{6}}-1
\end{aligned}
$$


$\Rightarrow \psi_{2}(s)=\frac{1}{p_{2}(s)}=\frac{1}{s^{\frac{5}{6}-1}}$

$\Rightarrow u_{2}(t)=\mathcal{L}^{-1}\left[\psi_{2}(s)\right]=t^{\frac{-1}{6}} E_{\frac{5}{6}, \frac{5}{6}}\left(t^{\frac{5}{6}}\right)$

Where the initial approximation must be satisfied by the following equations:

$$
\begin{aligned}
& L_{1} x(t)=0, x(0)=1,\left.\left({ }_{R L} D^{\alpha+\beta-1} x(t)\right)\right|_{t=0}= \\
& \left.\left({ }_{R L} D^{\frac{-1}{6}} x(t)\right)\right|_{t=0}=0,\left.\left({ }_{R L} D^{\beta-1} x(t)\right)\right|_{t=0}=\left.\left({ }_{R L} D^{\frac{-2}{3}} x(t)\right)\right|_{t=0}= \\
& 0 \Longrightarrow x_{0}(t)=E_{\frac{5}{6}, 1}\left(-t^{\frac{5}{6}}\right) \\
& L_{2} y(t)=0, y(0)=1,\left.\left(D^{\alpha+\beta-1} y(t)\right)\right|_{t=0}=\left.\left({ }_{R L} D^{\frac{-1}{6}} y(t)\right)\right|_{t=0}= \\
& 0,\left.\left({ }_{R L} D^{\beta-1} y(t)\right)\right|_{t=0}=\left.\left({ }_{R L} D^{\frac{-2}{3}} y(t)\right)\right|_{t=0}=0, y_{0}(t)=E_{\frac{5}{6}, 1}\left(t^{\frac{5}{6}}\right) \\
& z_{0}(t)=\sin t
\end{aligned}
$$

Accordingly, by Eq. (4.6) the higher order approximation of the exact solution can be obtained as follows:

$$
\begin{aligned}
& x_{1}(t)=\sum_{k=0}^{\infty} \frac{\left(-t^{\frac{5}{6}}\right)^{k}}{\Gamma\left(\frac{5}{6} k+1\right)}+ \\
& \int_{0}^{\tau}\left[( ( t - \tau ) ^ { \frac { - 1 } { 6 } } \sum _ { k = 0 } ^ { \infty } \frac { ( - ( t - \tau ) ^ { \frac { 5 } { 6 } } ) ^ { k } } { \Gamma ( \frac { 5 } { 6 } k + \frac { 5 } { 6 } ) } ) \left(2 \tau \sum_{k=0}^{\infty} \frac{(k+1) !}{k !} \frac{\left(\tau^{\frac{5}{6}}\right)^{k}}{\Gamma\left(\frac{5}{6} k+\frac{11}{6}\right)}-\right.\right. \\
& 2 \sum_{k=0}^{\infty} \frac{(k+1) !}{k !} \frac{\left(-\tau^{\frac{5}{6}}\right)^{k}}{\Gamma\left(\frac{5}{6} k+\frac{11}{6}\right)}-2 \tau \cos \tau+(4+2 \tau) \sum_{k=0}^{\infty} \frac{\left(\tau^{\frac{5}{6}}\right)^{k}}{\Gamma\left(\frac{5}{6} k+1\right)}- \\
& \left.\left.\left.\left(2 \tau^{2}+6 \tau\right) \sin \tau\right)\right]_{d \tau} d\right]^{k}\left[(t-\tau)^{\frac{-1}{6}} \sum_{k=0}^{\infty} \frac{\left((t-\tau)^{\frac{5}{6}}\right)^{k}}{\Gamma\left(\frac{5}{6} k+\frac{5}{6}\right)}\right)(\cos \tau- \\
& y_{1}(t)=\sum_{k=0}^{\infty} \frac{\left(t^{\frac{5}{6}}\right)^{k}}{\Gamma\left(\frac{5}{6} k+1\right)}+\int_{0}^{\tau}[( \\
& 2 \tau \sin \tau)] d \tau \\
& z_{1}(t)=\sin t
\end{aligned}
$$

Case 2: $\alpha+\beta=1\left(\right.$ if $\left.\alpha=\frac{2}{5}, \beta=\frac{6}{10}\right)$

$L_{1} x(t):{ }_{R L} D^{\frac{2}{3}}\left({ }_{R L} D^{\frac{6}{10}} x(t)\right)+x(t)$

$\Rightarrow p_{1}(s)=s+1$

$\Rightarrow \psi_{1}(s)=\frac{1}{p_{1}(s)}=\frac{1}{s+1}$

$\Longrightarrow u_{1}(t)=\mathcal{L}^{-1}\left[\psi_{1}(s)\right]=e^{-t}$

And

$$
\begin{aligned}
& L_{2} y(t):{ }_{R L} D^{\frac{2}{3}}\left({ }_{R L} D^{\frac{6}{10}} y(t)\right)-y(t) \\
& \Rightarrow p_{2}(s)=s-1 \\
& \Rightarrow \psi_{2}(s)=\frac{1}{p_{2}(s)}=\frac{1}{s-1} \\
& \Rightarrow u_{2}(t)=\mathcal{L}^{-1}\left[\psi_{2}(s)\right]=e^{t}
\end{aligned}
$$

Where the initial approximation must be satisfied by the following equations:
$L_{1} x(t)=0, x(0)=1,\left.\left({ }_{R L} D^{\beta-1} x(t)\right)\right|_{t=0}=\left.\left({ }_{R L} D^{\frac{-4}{10}} x(t)\right)\right|_{t=0}=$ $0 \Longrightarrow x_{0}(t)=e^{-t}$

$L_{2} y(t)=0, y(0)=1,\left.\left({ }_{R L} D^{\beta-1} y(t)\right)\right|_{t=0}=\left.\left({ }_{R L} D^{\frac{-4}{10}} y(t)\right)\right|_{t=0}=$ $0 \Rightarrow y_{0}(t)=e^{t}$

$z_{0}(t)=\sin t$

Accordingly, by Eq. (4.6) the higher order approximation of the exact solution can be obtained as follows:

$x_{1}(t)=e^{-t}+\int_{0}^{\tau}\left[\left(\sum_{k=0}^{\infty} \frac{(\tau-t)^{k}}{\Gamma(k+1)}\right)\left(2 \tau^{2} \sum_{k=0}^{\infty} \frac{(\tau)^{k}}{\Gamma(k+1)}-\right.\right.$ $2 \tau \sum_{k=0}^{\infty} \frac{(-\tau)^{k}}{\Gamma(k+1)}-2 \tau \cos \tau+(4+2 \tau) \sum_{k=0}^{\infty} \frac{(\tau)^{k}}{\Gamma(k+1)}-\left(2 \tau^{2}+\right.$ $6 \tau) \sin \tau)] d \tau$

$y_{1}(t)=e^{t}+\int_{0}^{\tau}\left[\left(\sum_{k=0}^{\infty} \frac{(t-\tau)^{k}}{\Gamma(k+1)}\right)(\cos \tau-2 \tau \sin \tau)\right] d \tau$ $z_{1}(t)=\sin t$

Case 3: $\alpha+\beta>1$ (if $\alpha=\frac{7}{12}, \beta=\frac{3}{4}$ )

$L_{1} x(t):{ }_{R L} D^{\frac{7}{12}}\left({ }_{R L} D^{\frac{3}{4}} x(t)\right)+x(t)$

$\Rightarrow p_{1}(s)=s^{\frac{4}{3}}+1$

$\Rightarrow \psi_{1}(s)=\frac{1}{p_{1}(s)}=\frac{1}{s^{\frac{4}{3}}+1}$

$\Rightarrow u_{1}(t)=\mathcal{L}^{-1}\left[\psi_{1}(s)\right]=t^{\frac{1}{3}} E_{\frac{4}{3}, \frac{4}{3}}\left(-t^{\frac{4}{3}}\right)$

And

$L_{2} y(t):{ }_{R L} D^{\frac{7}{12}}\left({ }_{R L} D^{\frac{3}{4}} y(t)\right)-y(t)$

$\Rightarrow p_{1}(s)=s^{\frac{4}{3}}-1$

$\Rightarrow \psi_{2}(s)=\frac{1}{p_{2}(s)}=\frac{1}{s^{\frac{4}{3}}-1}$

$\Rightarrow u_{2}(t)=\mathcal{L}^{-1}\left[\psi_{2}(s)\right]=t^{\frac{1}{3}} E_{\frac{4}{3}, \frac{4}{3}}\left(t^{\frac{4}{3}}\right)$

Where the initial approximation must be satisfied by the following equations:

$L_{1} x(t)=0, x(0)=1, \dot{x}(t)=0,\left.\left({ }_{R L} D^{\alpha+\beta-1} x(t)\right)\right|_{t=0}=$

$\left.\left({ }_{R L} D^{\frac{1}{3}} x(t)\right)\right|_{t=0}=0,\left.\left({ }_{R L} D^{\beta-1} x(t)\right)\right|_{t=0}=\left.\left({ }_{R L} D^{\frac{-1}{4}} x(t)\right)\right|_{t=0}=$

$\Rightarrow x_{0}(t)=E_{\frac{4}{3}, 1}\left(-t^{\frac{4}{3}}\right)$

$L_{2} y(t)=0, y(0)=1, y(t)=0,\left.\left({ }_{R L} D^{\alpha+\beta-1} y(t)\right)\right|_{t=0}=$ $\left.\left({ }_{R L} D^{\frac{1}{3}} y(t)\right)\right|_{t=0}=0,\left.\left({ }_{R L} D^{\beta-1} y(t)\right)\right|_{t=0}=\left.\left({ }_{R L} D^{\frac{-1}{4}} y(t)\right)\right|_{t=0}=$

$\Rightarrow y_{0}(t)=E_{\frac{4}{3}, 1}\left(t^{\frac{4}{3}}\right)$

$z_{0}(t)=\sin t$

Accordingly, by Eq. (4.6) the higher order approximation of the exact solution can be obtained as follows:

$x_{1}(t)=\sum_{k=0}^{\infty} \frac{\left(-t^{\frac{4}{3}}\right)^{k}}{\Gamma\left(\frac{4}{3} k+1\right)}+$ 


$$
\begin{aligned}
& \int_{0}^{\tau}\left[( ( t - \tau ) ^ { \frac { 1 } { 3 } } \sum _ { k = 0 } ^ { \infty } \frac { ( - ( t - \tau ) ^ { \frac { 4 } { 3 } } ) ^ { k } } { \Gamma ( \frac { 4 } { 3 } k + \frac { 4 } { 3 } ) } ) \left(2 \tau \sum_{k=0}^{\infty} \frac{(k+1) !}{k !} \frac{\left(\tau^{\frac{4}{3}}\right)^{k}}{\Gamma\left(\frac{4}{3} k+\frac{7}{3}\right)}-\right.\right. \\
& 2 \sum_{k=0}^{\infty} \frac{(k+1) !}{k !} \frac{\left(-\tau^{\frac{4}{3}}\right)^{k}}{\Gamma\left(\frac{4}{3} k+\frac{7}{3}\right)}-2 \tau \cos \tau+(4+2 \tau) \sum_{k=0}^{\infty} \frac{\left(\tau^{\frac{4}{3}}\right)^{k}}{\Gamma\left(\frac{4}{3} k+1\right)}- \\
& \left.\left.\left(2 \tau^{2}+6 \tau\right) \sin \tau\right)\right] d \tau \\
& y_{1}(t)=\sum_{k=0}^{\infty} \frac{\left(t^{\frac{4}{3}}\right)^{k}}{\Gamma\left(\frac{4}{3} k+1\right)}+\int_{0}^{\tau}\left[\left((t-\tau)^{\frac{1}{6}} \sum_{k=0}^{\infty} \frac{\left((t-\tau)^{\frac{4}{3}}\right)^{k}}{\Gamma\left(\frac{4}{3} k+\frac{4}{3}\right)}\right)(\cos \tau-\right. \\
& 2 \tau \sin \tau)] d \tau \\
& z_{1}(t)=\sin t
\end{aligned}
$$

\begin{tabular}{|c|c|c|c|c|}
\hline $\mathrm{T}$ & $\begin{array}{c}\mathrm{x}_{1} \\
\alpha=\frac{1}{2}, \beta=\frac{1}{3}\end{array}$ & $\begin{array}{c}\mathrm{x}_{1} \\
\alpha=\frac{2}{5}, \beta=\frac{6}{10}\end{array}$ & $\begin{array}{c}x_{1} \\
\alpha=\frac{7}{12}, \beta=\frac{3}{4}\end{array}$ & Exact solution \\
\hline 0 & 125 & 120 & 1 & 1 \\
\hline 0.1 & 1.366 & 1.313 & 1.094 & 1.015 \\
\hline 0.2 & 1.755 & 1.647 & 1.242 & 1.063 \\
\hline 0.3 & 1.796 & 1.75 & 1.391 & 1.146 \\
\hline 0.4 & 1.983 & 1.9 & 1.555 & 1.267 \\
\hline 0.5 & 2.125 & 1.982 & 1.711 & 1.431 \\
\hline 0.6 & 2.22 & 1.988 & 1.846 & 1.642 \\
\hline 0.7 & 2.457 & 2.212 & 1.949 & 1.906 \\
\hline 0.8 & 2.761 & 2.549 & 2.341 & 2.23 \\
\hline 0.9 & 3.117 & 2.903 & 2.674 & 2.62 \\
\hline 1 & 3.852 & 3.561 & 3.196 & 3.086 \\
\hline
\end{tabular}

Table 2: Numerical Results of the Solution in Example 2

Table 2 shows the approximate solutions for Eq. (4.6) obtained for different values of $\alpha$ and $\beta$ using our method. The results are in good agreement with the results of the exact solutions.

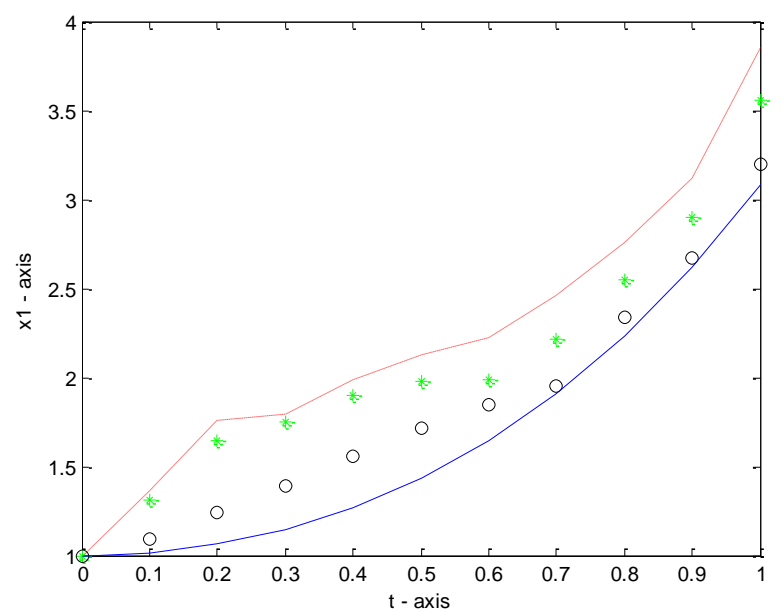

Fig. 2: The red dotted line (..) is the approximate solution when $\alpha=$ $\frac{1}{2}, \beta=\frac{1}{3}(\alpha+\beta<1)$, the green asterisks $(* * *)$ is the approximate solution when $\alpha=\frac{2}{5}, \beta=\frac{6}{10}(\alpha+\beta=1)$, the black circles (ooo) is the approximate solution when $\alpha=\frac{7}{12}, \beta=\frac{3}{4}(\alpha+\beta>1)$, the continuous line (---) is the exact solution.

\section{Conclusions}

The fundamental goal of this work has been to propose an efficient algorithm for the solution of non-homogeneous system of time-fractional differential-algebraic equations. The goal has been achieved by using the fractional Laplace iteration method (FLIM).The results show that fractional Laplace iteration method is powerful and efficient techniques in finding approximate solu- tions for a system of time-fractional linear differential-algebraic equations Mathcad has been used for computations in this paper.

\section{Acknowledgement}

The authors express their sincere thanks to the referees for their careful review of this manuscript and their useful suggestions which lead to an improved version.

\section{References}

[1] R. Hilfer, "Applications of fractional calculus in physics", World Scientific, Singapore, 2000. https://doi.org/10.1142/3779.

[2] R. Gorenflo, F. Mainardi, "Fractional calculus: Integral and differential equations of fractional order, in: A. Carpinteri, F. Mainardi (Eds.), Fractals and Fractional Calculus in Continuum Mechanics", Springer, New York, (1997) 223-276. https://doi.org/10.1007/9783-7091-2664-6 5.

[3] A. Carpinteri, F. Mainardi, "Fractals and Fractional Calculus in Continuum Mechanics", Springer Verlag, Wien, New York, (1997). https://doi.org/10.1007/978-3-7091-2664-6.

[4] F. Mainardi, "Fractional caculus: Some basic problem in continuum and statistical mechanics" in A. Carpinteri, F. Mainardi (Eds.), Fractals and Fractional Calculus in Continuum Mechanics, Springer, New York, (1997) 291-348. https://doi.org/10.1007/978-3-70912664-6 7 .

[5] U. M. Ascher, L R. Petzold, "Projected implicit Runge-Kutta methods for differential-algebraic equations", SIAM Journal on Numerical Analysis, $28 \quad$ (1991) 1097-1120. https://doi.org/10.1137/0728059

[6] N. Guzel, M. Bayram, "On the numerical solution of differentialalgebraic equations with index-3", Appl. Math. Comput, 175 (2) (2006) 1320-1331. https://doi.org/10.1016/j.amc.2005.08.025.

[7] N. Guzel, M. Bayram, "Numerical solution differential-algebraic equations with index-2", Appl. Math. Comput., 174 (2) (2006) 1279-1289. https://doi.org/10.1016/j.amc.2005.05.035.

[8] W. Wang, "An algorithm for solving DAEs with mechanization", Appl. Math. Comput., 167 (2) (2005) 1350-1372. https://doi.org/10.1016/j.amc.2004.08.010.

[9] E. Celik, M. Bayram, "The numerical solution of physical problems modeled as a systems of differential-algebraic equations (DAEs)", J. Franklin Inst., $342 \quad$ (1) (2005) 1-6. https://doi.org/10.1016/j.jfranklin.2004.07.004.

[10] S. Momani, Z. Odibat, "Numerical approach to differential equations of fractional order", J. Comput. Appl. Math., 207 (2007) 96110. https://doi.org/10.1016/j.cam.2006.07.015.

[11] S. Momani, Z. Odibat, "Homotopy perturbation method for nonlinear partial differential equation of fractional order", Phys. Lett. A 365 https://doi.org/10.1016/j.physleta.2007.01.046.

[12] Z. Odibat, S. Momani, "Modified homotopy perturbation method Application to quadratic Riccati differential equation of fractional order", Chaos. Solitions Fractals, 36(1) (2006) 167-174. https://doi.org/10.1016/j.chaos.2006.06.041.

[13] F. Soltanian, Mehdi .Dehghan, S. M. Karbasi, "Solution of the differential algebraic equations via homotopy perturbation method and their engineering applications", International Journal of Comput. $\begin{array}{lrr}\text { Math., } & 9 & \text { (2010) }\end{array}$ https://doi.org/10.1080/00207160802545908.

[14] B. Barani, S.M. Hosseini, M. Saffarzadeh, S. Javanmardi, "Analytical approach of differential-algebraic equation of frational order via homotopy perturbation method", Communications on Advanced Compt. Scien. With Appli., 2014 (2014) 1-8.

[15] M. M. Hosseini, "Adomian decomposition method for solution of differential algebraic equations", Journal of Comput. and Appl. Math., $\quad 197 \quad$ (2006) 495-501 https://doi.org/10.1016/j.cam.2005.11.012.

[16] M. Hosseini, "Adomian decomposition method for solution of nonlinear differential algebraic equations", Appl. Math. And Comput., 181 (2006) 1737-1744. https://doi.org/10.1016/j.amc.2006.03.027.

[17] E. Celike, M. Bayramb, T. Yelogu, "Solution of differentialalgebraic equations (ADEs) by Adomian decomposition method", Inter. J. Pure Appl. Math. Sci., 3(1) (2006) 93-100.

[18] S. Momani, K. Al. Khaled, "Numerical solution for system of fractional differential equations by the decomposition method", Appl. Math. Comput., $162 \quad$ (2005) 1351-1365. https://doi.org/10.1016/j.amc.2004.03.014. 
[19] H. Jafari, V. D. Gejji, "Solving a system of nonlinear fractional equations usingf Adomian decomposition", Appl. Math. Comput. 196 (2006) 644-651. https://doi.org/10.1016/j.cam.2005.10.017.

[20] S. J. Liao, "The proposed homotopy analysis technique for the solution of nonlinear problems", Ph. D. Tesis, Shanghnai Jiao Tong University, 1992.

[21] M. Zurigat, S. Momani, A. Alawneh, "Analytical approximate solution of systems of fractional algebraic- differential equations by homotopy analysis method", Comput. And Math. With Appl., 59 (2010) 1227-1235. https://doi.org/10.1016/j.camwa.2009.07.002.

[22] F. Soltanian, S. M. Karbasi, M. M. Hosseini, "Application of He' variational iteration method for solution of differential-algebraic equations", Chaos, Sloitons and Fractals, 41(1) (2004) 436-445. https://doi.org/10.1016/j.chaos.2008.02.004.

[23] Z. Odibat, S.Momani, "Application of variation iteration method to nonlinear differential equations of fractional order", Int. Nonlin. Sci. Numer. Simulat., 1 (7) (2006) 15-27.

[24] S. Momani, Z. Odibat, "Numerical comparsion of methods for solving linear differential equation of fractional order", Chaos Solitons Fractals, $\quad 31 \quad$ (2007) https://doi.org/10.1016/i.chaos.2005.10.068.

[25] S. Gupta, D. Kumar, J. Singh, "Numerical study for systems of fractional differential equations via Laplace transform", Journal of the Egypian Math. Society, 23 (2015) 256-262.

[26] H. Latifizadeh, E. Hesameddini,"Flexibility and efficiency of new analytical method for solving systems of linear and nonlinear differential equations", International Journal of Science and Engineering Investigations", 2 ( July 2013) 44-51.

[27] I. Podlubny, "Fractional differential equations", New York, NY: A cademic Press, 1999.

[28] S. Kazem, "Exact solution of some linear fractional differential equations by laplace transform", Tnternational Journal of Nonlinear Science, 16 (1) (2013) 3-11. 